\title{
Reflets
}

Revue ontaroise d'intervention sociale et communautaire

\section{Bibliographie des articles arbitrés du Dossier - 1995 à 2006}

Volume 13, numéro 1, 2007

La violence dans tous ses états

URI : https://id.erudit.org/iderudit/016818ar

DOI : https://doi.org/10.7202/016818ar

Aller au sommaire du numéro

Éditeur(s)

Reflets : Revue ontaroise d'intervention sociale et communautaire

ISSN

1203-4576 (imprimé)

1712-8498 (numérique)

Découvrir la revue

Citer ce document

(2007). Bibliographie des articles arbitrés du Dossier - 1995 à 2006. Reflets,

13(1), 202-207. https://doi.org/10.7202/016818ar

Tous droits réservés (C) Reflets : Revue ontaroise d'intervention sociale et communautaire, 2007
Ce document est protégé par la loi sur le droit d'auteur. L'utilisation des services d'Érudit (y compris la reproduction) est assujettie à sa politique d'utilisation que vous pouvez consulter en ligne.

https://apropos.erudit.org/fr/usagers/politique-dutilisation/ 


\section{Bibliographie des articles arbitrés du Dossier - 1995 à 2006}

\section{Volume 1, numéro 1}

DIALLO, Lamine et Marge REITSMA-STREET (1995). "Stratégies de survie et d'identité : les dynamiques culturelles dans un projet d'intervention en prévention communautaire ", printemps, 43-69.

GÉLINAS, Mirelle (1995). "Réflexions des thérapeutes familiaux francophones de la région d'Ottawa-Carleton sur la famille franco-ontarienne ", printemps, 70-91.

MOLGAT, Marc avec la collaboration de Denise Lemire (1995). «Des jeunes à l'écart du social? Histoires de jeunes francophones sans abri à Ottawa-Carleton ", printemps, 141-163.

MVILONGO-TSALA, Anselme (1995). "Formation et intervention interculturelle : défi pour l'Ontario français ", printemps, 122-140.

NDUWIMANA, Mathilde et Alice HOME (1995). «Vers des services attirants, accessibles et pertinents pour les personnes âgées immigrantes francophones? ", printemps, 92-121.

WELCH, David (1995). "Les Franco-Ontariens : la résistance comme mode de vie ", printemps, 20-42.

\section{Volume 1, numéro 2}

ADAM, Dyane (1995). «Étude comparative des caractéristiques internes et de l'environnement interne et de l'environnement externe des communautés d'entraide francophone et anglophone de Sudbury-Manitoulin ", automne, 72-89.

BARETTE, Lise, PICARD, Louise, BEYERS, Joanne et Teresa TAILLEFER (1995). « Programme de promotion de la santé et du bien-être au travail : le travail par quarts ... ça s'améliore ", automne, 115-137.

BLAIS, Louise (1995). «Étiologie sociale et santé mentale : ouvertures et fermetures des modèles explicatifs dominants ", automne, 138-162.

CODERRE, Cécile (1995). "Femmes et santé, en français s'il vous plaît ", automne, 38-71.

GAGNÉ, Elsy (1995). "Les chemins qu'on parcourt pour livrer un combat contre la maladie sont très difficiles par bouts. La réalité des Franco-Manitobaines et le cancer du sein ", automne, 90-114. 


\section{Volume 2, numéro 1}

de LAJUDIE, Martine (1996). "Le secret et l'enfant victime de maltraitance sexuelle ", printemps, 69-95.

DUHAMEL-MAPLES, Marthe (1996). «La violence en milieu scolaire : un défi pour intervenants et intervenantes ", printemps, 40-57.

TROCMÉ, Nico (1996). «L'étranger : ogre ou bon samaritain? », printemps, 58-68.

\section{Volume 2, numéro 2}

GARCEAU, Marie-Luce (1996). "Bénévolat des femmes vieillissantes à l'aube de l'an 2000 ", automne, 58-81.

LADOUCEUR, Lisa (1996). " Différence chez les personnes âgées : mise en contexte ", automne, 82-97.

SIMARD, Noël (1996). « Dilemmes éthiques des soins aux personnes âgées », automne, 38-57.

\section{Volume 3, numéro 1}

DROLET, Marie (1997). «L'empowerment et intervention familiale : concept paradoxal occultant parfois la pauvreté ", printemps, 55-79.

KÉRISIT, Michèle et Nérée ST-AMAND (1997). « Résistance et créativité : pratiques alternatives des familles démunies ", printemps, 30-54.

MONGRAIN, Manon (1997). "Cuisines collectives : modèle alternatif d'organisation sociale et économique ", printemps, 80-100.

\section{Volume 3, numéro 2}

BERGER, Marie-Josée (1997). "À la recherche d'une intégration : rêve ou réalité? ", automne, 74-86.

BERNIER, Christiane (1997). «Conviction de la différence : synthèse d'une recherche sur la famille francophone en Ontario ", automne, 87-105.

BERNIER, Christiane et Renée C. MALLET (1997). "Les jeunes et leurs visions du féminisme ", automne, 128-142.

CÔTÉ, Andrée (1997). "Éducation juridique populaire sur les droits des femmes en Ontario ", automne, 50-73.

SEMBLAT, Marie-Lise (1997). "Européennes, “ actrices actives" de développement rural. Structuration de nouveaux groupes de femmes ", automne, 106-127. 


\section{Volume 4, numéro 1}

BEAUSOLEIL, Natalie (1998). «Corps, santé, apparence et vieillissement dans les énoncés de femmes francophones en Ontario ", printemps, 53-74.

CHOLETTE, Chantal (1998). "Le travail obligatoire en Ontario : solution ou imposture?", printemps, 100-126.

KÉRISIT, Michèle (1998). "Les défis de l'intervention interculturelle en milieu minoritaire de langue française on Ontario ", printemps, 75-99.

MIANDA, Gertrude (1998). «Être une immigrante noire africaine francophone à Toronto : vécu et perception des rapports de genre ", printemps, 34-52.

\section{Volume 4, numéro 2}

BARETTE, Jacques, LAROCHE, Chantal et Linda J. GARCIA (1998). «Troubles de la communication et obstacles au travail : les défis des personnes et des organisations ", automne, 47-62.

BLAIS, Louise et Louise MULLIGAN-ROY (1998). "Incapacité mentale et incapacité sociales ", automne, 87-103.

BURELLE, Roxanne (1998). "L'intégration des personnes ayant une incapacité, un avenir à créer ", automne, 32-46.

CHARRON, Marc (1998). "Sourds ou malentendants, une question de culture? ", automne, 63-86.

\section{Volume 5, numéro 1}

BAGAOUI, Rachid et Donald A.DENNIE (1999). «Le développement économique communautaire : nouveau départ pour le mouvement associatif Franco-Ontarien? ", printemps, 75-94.

LEDUC BROWNE, Paul (1999). "La dialectique de l'économie sociale : travail, employabilité, solidarité ", printemps, 28-53.

MICHAUD, Jacinthe (1999). «Les femmes francophones et le travail obligatoire : un enjeu pour l'économie sociale ", printemps, 95-113.

WELCH, David (1999). "L'économie sociale en Ontario français : analyse historique, pratiques actuelles et recherche de sens ", printemps, 54-74.

\section{Volume 5, numéro 2}

BOUCHARD, Lyne et Linda CARDINAL (1999). "Conditions de possibilités des services en français en Ontario dans les domaines de la santé et des services sociaux : un enjeu pour les femmes ", automne, 109-122.

BOUDREAU, Françoise (1999). "Langue minoritaire et services de santé mentale en l'an 2000 : droits et besoins des francophones de Toronto ", automne, 123-154. 
BOUDREAU, Françoise et Diane FARMER (1999). "Profil épidémiologique des francophones de l'Ontario : les faits saillants revisités et comparés ", automne, 103-108.

PICARD, Louise et Janine CHARLAND (1999). «Le profil démographique et les déterminants de la santé des francophones en Ontario ", automne, 44-63.

PICARD, Louise et Denise HÉBERT (1999). «L'état de santé de la population francophone de l'Ontario ", automne, 64-102.

\section{Volume 6, numéro 1}

LECLERC, Robert (2000). "Services en santé mentale pour les personnes ayant un handicap intellectuel : combler des lacunes ", printemps, 90-106.

ST-AMAND, Nérée (2000). «Des noms qui en disent long... " "printemps, 36-63.

VAN DE SANDE, Adje et Jean-Marc BÉLANGER (2000). "La pauvreté chez les enfants et les jeunes de Sudbury : une approche d'intervention à l'échelle régionale ", printemps, 64-89.

\section{Volume 6, numéro 2}

CODERRE, Cécile et Madeleine DUBOIS (2000). "Solidarité et citoyenneté : initiatives pour contrer la pauvreté chez les francophones dans Ottawa-Carleton », automne, 61-86.

CÔTÉ, Geneviève M. (2000). «Les pertes, les deuils et le processus de croissance des femmes ", automne, 117-137.

GÉROME, Marie-Hélène (2000). " Le travail des soins à domicile en Ontario, creuset de nouvelles formes de discrimination à l'endroit des femmes? ", automne, 87-116.

MICHAUD, Jacinthe (2000). «La politique du workfare en Ontario :les groupes de femmes coincés entre la nécessité et l'État néolibéral ", automne, 34-60.

MUJAWAMARIYA, Donatille (2000). "Ils ne peuvent pas enseigner dans nos écoles : le dilemme des étudiants-maitres des minorités visibles nés au Canada ", automne, 138-165.

\section{Volume 7, numéro 1}

GAGNON, Julie (2001). "La loi 68 : loi en l'honneur de Brian Smith », printemps, 26-47.

JENKINS, Mary Ann,VAN DE SANDE, Adje et Jean-Marc BÉLANGER (2001). "L'Ontario au travail et son programme EXPRESS : une analyse critique ", printemps, 90-112.

KAUPPI, Carol, BÉLANGER, Jean-Marc et Jennifer KECK (2001). "Les sans-abri et la maladie mentale : perspectives des fournisseurs de services de Sudbury ", printemps, 48-69.

MVILONGO, Anselme et Michel-André BEAUVOLSK (2001). "Santé mentale et suicide des jeunes ", printemps, 70-89. 


\section{Volume 7, numéro 2}

ST-AMAND, Nérée (2001). "Dans l'ailleurs et l'autrement : pratiques alternatives et service social ", automne, 30-74.

\section{Volume 8, numéro 1}

DUBOULOZ, Claire-Jehanne, avec la collaboration de Josée Vallerand, Claude Lachaine, Anne Castonguay, Céline Gingras et Rosalyn Rabow (2002). "Exploration d'un processus de transformation des perspectives de sens chez un groupe de quatre personnes atteintes de la sclérose en plaques : la définition de soi ", printemps, 28-46.

PARADIS, JoAnne, DESROCHERS, Alain et Linda GARCIA (2002). «Le Groupe de recherche sur l'évaluation des troubles de la communication : historique, objectifs et réalisations ", printemps, 61-72.

ROBERTS, Patricia M. (2002). "Défis dans l'évaluation du bégaiement chez les bilingues ", printemps, 47-60.

\section{Volume 9, numéro 1}

BERNIER, Christiane (2003). "Ménopause et mitan de vie : deux phénomènes, une symbolique ", printemps, 110-147.

DUPONT, Natalie et Christiane BERNIER (2003). "Politiques contre le harcèlement sexuel. Comparaison et perception des agents et des plaignantes ", printemps, 53-79.

NAÏR, Nadia (2003). "Globalisation, délocalisation des entreprises et travail des femmes. L'exemple de l'entreprise hollandaise T.K. Fish à Tétouan, Maroc ", printemps, 22-52.

ST-ONGE, Renée (2003). «Fusion d'hôpitaux au Nord-est de l'Ontario : quel espace pour la parole des infirmières?", printemps, 80-109.

\section{Volume 9, numéro 2}

DROLET, Marie et Denise OUELlETTE (2003). "Enjeux et pratique sociale en SAE : les intervenantes seront-elles les exclues de leur propre pratique? ", vol. 9, no. 2, automne, 87-113.

ELIEV, Sika et Christiane BERNIER (2003). "Perceptions de femmes cadres dans une entreprise typiquement masculine ", vol. 9, no. 2, automne, 87-113.

GARCEAU, Marie-Luce (2003). "Quand le harcèlement se mêle au travail ou aux études... ", vol. 9 , no. 2 , automne, 58-86.

HOME, Alice et Stéphanie PEARCE (2003). «Triple journée de travail des mères d'enfants ayant une incapacité invisible", vol. 9, no. 2, automne, 163-185.

HOULE, Pascale (2003). «Les obstacles à l'intégration au marché du travail des femmes monoparentales à faible revenu ", vol. 9 , no. 2 , automne, 34-57.

ST-AMAND, Nérée (2003). "Interventions opprimantes ou conscientisantes? ", vol. 9, no.2, 139-162. 


\section{Volume 10}

GAUDET, Stéphanie et Catherine BOUCHARD (2004). « Intervenir auprès des jeunes de quartiers défavorisés : étude de cas du Centre de jeunes de la Basse-Ville d'Ottawa », 85-98.

GRELL, Paul (2004). «Représentations des jeunes précaires à propos de leurs pratiques dans le monde du travail et de la vie quotidienne ", 46-62.

LEBLANC, Patrice (2004). «Au-delà de l'argent et de l'emploi : stratégies d'intervention quant à la migration des jeunes non-métropolitains ", 63-84.

\section{Volume 11}

BRUCKERT, Christine et Colette PARENT (2005). « Répondre aux besoins des travailleuses du sexe de rue : un objectif qui passe par la décriminalisation de leurs activités de travail ", 112-145.

GILBERT,Anne, KÉRISIT, Michèle, DALLAIRE, Christine, CODERRE, Cécile et Jean HARVEY (2005). « Les discours sur la santé des organismes franco-ontariens : du rapport Dubois à la cause Montfort ", 20-48.

LABIDI, Lassaad (2005). "Quand l'exclusion intervient avec la retraite : l'expérience tunisienne ", 81-111.

MADIBBO, Amal et Normand LABRIE (2005). «La transformation des institutions et des communautés francophones face à l'immigration et à la mondialisation : une étude de cas ", 49-80.

\section{Volume 12}

CADELL, Susan et Dennis HAUBRICH (2006). «Vivre intensément l'humain : une exploration du deuil après le VIH-sida ", 127-143.

KHALID, Mohammed (2006). "Service social, spiritualité, vieillissement : de la négociation au dialogue interactif ", 74-106.

LAVOIE, Louis-Charles (2006). "Psychothérapie et spiritualité », 48-73.

OUELLETTE, Pierre et Dom Raymond CARETTE (2006). « Les pourquoi d'une retraite à l'Abbaye Saint-Benoît-du-Lac », 144-166.

SIMARD, Noël (2006). «Spiritualité et santé », 107-126.

ST-AMAND, Nérée (2006). "La spiritualité : au cœur ou en marge de l'intervention sociale?", $20-47$. 